\title{
The role of verbal and performance intelligence in children's strategy selection and execution
}

\author{
Koen Luwel \\ Ageliki Foustana \\ Patrick Onghena \\ Lieven Verschaffel
}
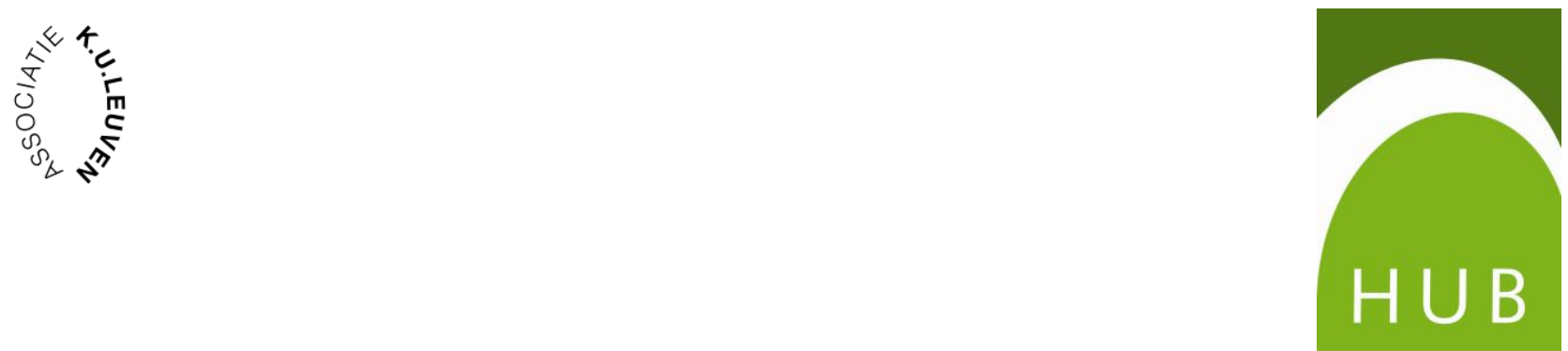


\section{RUNNING HEAD: INTELLIGENCE AND STRATEGIC PERFORMANCE}

The role of verbal and performance intelligence in children's strategy selection and

$$
\text { execution }
$$

\section{Koen Luwel*}

Centre for Educational Research and Development, Hogeschool-Universiteit Brussel

Stormstraat 2, B-1000 Brussel, Belgium

Koen.Luwel@hubrussel.be

Tel: $++32-2-6098856$

Centre for Instructional Psychology and Technology, KU Leuven

Vesaliusstraat 2, B-3000 Leuven, Belgium

$$
\text { Ageliki Foustana }
$$

Department of Special Education and Psychology, University of Athens

Harilaou Trikoupi 24, Athens, Greece

E-mail: afousta@primedu.uoa.gr

Patrick Onghena

Centre for Methodology of Educational Research, KU Leuven

Vesaliusstraat 2, B-3000 Leuven, Belgium

E-mail: Patrick.Onghena@ppw.kuleuven.be

Lieven Verschaffel

Centre for Instructional Psychology and Technology, KU Leuven

Vesaliusstraat 2, B-3000 Leuven, Belgium

E-mail: Lieven.Verschaffel@ppw.kuleuven.be

${ }^{*}$ Corresponding author 


\begin{abstract}
The present study investigated the extent to which verbal intelligence (VIQ) and performance intelligence (PIQ) contribute to strategy selection and execution in the context of a numerosity judgement task. The choice/no-choice method was used to appropriately assess strategy selection (in terms of strategy repertoire, frequency and, adaptivity) and strategy execution (in terms of strategy speed and accuracy) in a group of 120 12-year-old children. For each parameter, a regression analysis was carried out with VIQ and PIQ, as independent variables, and with arithmetic proficiency as a control variable. Results showed that VIQ was a significant predictor for all parameters of strategic competence, whereas PIQ only explained a unique portion of the variance above and beyond VIQ in the speed of strategy execution.
\end{abstract}

Keywords: strategy choice; strategy use; verbal intelligence; performance intelligence; choice/no-choice method 


\section{Introduction}

The research on the selection and execution of strategies has made a great progress in many domains of human cognition in the last 20 years (Siegler, 1996, 2005). Some time ago, Siegler and Lemaire (1997; see also Luwel, Onghena, Torbeyns, Schillemans, \& Verschaffel, 2009) advocated the choice/no-choice method as an ideal means to investigate the selection and execution of strategies in cognitive tasks. This method tests participants under two types of conditions: (a) a choice condition in which one can freely choose which strategy to use from a set of available strategies, and (b) a number of no-choice conditions in which one has to use one specific strategy. The choice data provide information about participants' strategy repertoire and strategy frequencies. The no-choice data provide unbiased measures of strategy speed and accuracy. Finally, a comparison of the actual strategy choices from the choice condition with an optimal pattern of strategy choices derived from the no-choice data allows assessing the adaptivity of strategy choices. Strategy selection typically involves the parameters of repertoire, frequency, and adaptivity, whereas strategy execution is typically analyzed in terms of speed and accuracy.

Although several studies have already investigated intelligence-related differences in children's strategic functioning (e.g., Gaultney, Bjorklund, \& Goldstein, 1996; Geary \& Brown, 1991; Hettinger-Steiner, 2006), none of these earlier investigations examined all parameters of strategic competence within a single task, nor did they asses these parameters by means of the choice/no-choice method. Recently, Luwel, Foustana, Papadatos, and Verschaffel (2011) applied this method to study the role of intelligence in children's strategic competence. More specifically, they compared the strategic competence of a group of low-, medium, and high-intelligent children in a task in which children had to 
determine different numerosities of green blocks that were presented in a grid. Two strategies were allowed to solve the different problems of the task: (a) an addition strategy, in which the (groups of) coloured blocks are added to determine the total numerosity, and (b) a subtraction strategy, in which the number of empty squares is subtracted from the total number of squares in the grid. The choice of these strategies was strongly dependent on the ratio of coloured blocks to empty squares in the grid: the addition strategy is especially applied on trials with few blocks and many empty squares, whereas the subtraction strategy was preferentially used on items with many blocks and few empty squares (see Figure 1). Luwel et al. (2011) demonstrated, among other things, that intelligence played a significant role in all parameters of strategic competence: with increasing intelligence, children were more likely to use an insightful strategy, to apply this strategy more frequently and efficiently and to select it more adaptively.

\section{[INSERT FIGURE 1 ABOUT HERE]}

The present study aims at elaborating the findings of Luwel et al. (2011) in two different ways by re-analyzing their original data. First, now that it is shown that intelligence - in terms of the WISC-III full scale IQ score - plays a role in all parameters of strategic competence, we wanted to examine its contribution in greater detail by distinguishing between verbal intelligence (VIQ) and performance intelligence (PIQ). This allows examining the extent to which verbal and visuo-spatial abilities would play a different role in the strategic competence in this task. 
Second, Luwel et al. (2011) compared three different intelligence groups. Although this approach is widely used in the study of intelligence-related effects on the use of cognitive strategies (e.g., Geary \& Brown, 1991; Wong, 1982), it does not use all the variability in the sample since all participants within a specific intelligence range are treated as being equal in terms of their intelligence. In the present study, we treated VIQ and PIQ as continuous, rather than as discrete variables to sketch a more fine-grained picture of the role of intelligence in individuals' strategic competence. Moreover, the present analytical approach also allows assessing the unique contribution of both intelligence types to each of the different competence parameters.

2. Method

\subsection{Participants}

Participants were 120 seventh-grade students (52 boys and 68 girls) from several private and public schools in the county of Attica (Greece). Their mean chronological age was 12.54 yrs. ( $S D=.36$ yrs., range: 11.41 to $13.67 \mathrm{yrs})$.

\subsection{Material}

\subsubsection{Intelligence test}

Children's intelligence was assessed by means of the standardised Greek version of the WISC-III (Georgas, Paraskevopoulos, Bezevegis, \& Giannitsas, 1997; Wechsler, 1991). Their full scale IQ indicated that this sample covered the intended broad intelligence range (see Table 1) The VIQ-score is based on the composite score of the five verbal subtests of the WISC-III (i.e., information, similarities, arithmetic, vocabulary, and comprehension), whereas the PIQ-score is derived from the composite score of the WISC-III's five (visuospatial) performance subtests (i.e., picture completion, coding, picture arrangement, block 
design, and object assembly). The descriptive statistics of the different measures can be found in Table 1.

\subsubsection{Arithmetic proficiency test}

Since arithmetic proficiency may play a prominent role in the efficiency of the subtraction strategy, we included it as a control variable in our analyses. Children's arithmetic proficiency was assessed on the basis of the addition and the subtractionmultiplication subtests of the French Kit (French, Ekstrom, \& Price, 1963) which measure of the arithmetic skills underlying the use of the subtraction strategy more precisely than the arithmetic subtest of the WISC-III.

\subsubsection{Numerosity judgement task}

The numerosity judgement task was presented on a PC. Stimuli were square grids consisting of $7 \times 7$ little square units of $1 \times 1 \mathrm{~cm}$ each. We chose the relatively small $7 \times 7$ grid to ensure that all participants could solve all trials relatively easily by solely using the addition or the subtraction strategy and thus had not to resort to guessing (Luwel, Lemaire, \& Verschaffel, 2005). These square units could either be “on” (i.e., being filled with a green coloured block) or "off” (remaining empty, i.e., having the same black colour as the background of the whole of the screen). Participants ran 26 trials whereby all numerosities of green blocks between 20 and 45 were presented. This is the numerosity range in which children of this age group typically switch from the addition towards the subtraction strategy (e.g., Luwel et al., 2005). For each participant, the sequence of the stimuli as well as the placement of the blocks in the grid was randomised by the computer.

[INSERT TABLE 1 ABOUT HERE] 


\subsection{Procedure}

All participants were tested individually in two separate test sessions. During the first test session, they were administered the WISC-III and the arithmetic tests, whereas in the second session they accomplished the numerosity judgement task in a choice/no-choice design. The numerosity judgement task started with five example trials that were representative for the whole numerosity continuum. Participants were instructed to determine each of the numerosities as accurately and fast as possible and to indicate with their finger the green blocks/empty squares they were counting. This instruction allowed the experimenter to determine in a reliable and non-obtrusive way which strategy participants were applying.

All participants ran the choice condition first, to avoid that their strategy choices in the choice condition could be affected by carry-over effects from the no-choice conditions (Siegler \& Lemaire, 1997). Instructions at the beginning of the choice condition depended upon children having used the subtraction strategy during the practice trials or not. If children had shown evidence for the use of the addition and the subtraction strategy during the practice trials, they were told that they could use both strategies. However, if they only had applied the addition strategy during the practice trials, the experimenter only referred to that strategy in her further instruction since we expected that mentioning the subtraction strategy would have disturbed participants' natural strategy behavior in that condition. However, as soon as these participants spontaneously started to apply the subtraction strategy, the experimenter indicated that this strategy was also acceptable. 
In the two no-choice conditions, participants were told that they had to determine all numerosities of blocks by using only one strategy, either the addition strategy (in the nochoice/addition condition) or the subtraction strategy (in the no-choice/subtraction condition). The presentation order of the different conditions was counterbalanced across participants.

Stimuli remained on the screen until participants had made their numerosity judgement. They were asked to verbally state their answer as soon as they knew it. The experimenter then immediately pressed a key that stopped the computer timer and emptied the grid at the same time. After each trial, the experimenter entered participants' response and strategy, after which a new stimulus appeared on the screen. A brief pause was given between the different conditions.

\section{Results}

Since the subtraction strategy can be regarded as a "clever" strategy for solving the large-numerosity items of this task, we expected that the influence of VIQ and PIQ would be largest on its selection and execution. Our analyses therefore focused on the subtraction strategy.

We conducted a series of (logistic) regression analysis to explore the possible contribution of VIQ and PIQ to the different strategic competence parameters of the subtraction strategy, while including arithmetic proficiency as a control variable. Before carrying out these regression analyses, we first looked at the zero-order correlations between the different variables involved in this study (see Table 2). It can be seen that the different predictors show strong correlations with the different measures of strategic competence, which all go in the expected direction: the higher one scores on each of the 
predictors, the more frequently one uses the clever subtraction strategy, the lower its execution time and error rate, and the more adaptively it is selected.

\section{[INSERT TABLE 2 ABOUT HERE]}

We tested for the presence of multicollinearity by means of the 'Variance Inflation Factor' (VIF). The VIFs were 3.27, 2.64, and 1.62 for VIQ, PIQ, and arithmetic proficiency, respectively. Given that it is commonly accepted that VIFs less than 10 indicate acceptable multicollinearity (Neter, Kutner, Nachtsheim, \& Wasserman, 1996), multicollinearity was not an issue here.

\subsection{Strategy selection}

Fifty-nine out of 120 participants (i.e., 49\%) had the subtraction strategy in their repertoire (i.e., they applied the subtraction strategy at least once on the different items the choice condition). The binary logistic regression on the presence of the subtraction strategy in the strategic repertoire yielded a statistically significant model, $\chi^{2}(3)=89.01, p<.001$, indicating that it was able to distinguish between participants who did and did not possess the subtraction strategy. This model explained between 52\% (Cox and Snell $R^{2}$ ) and 70\% (Nagelkerke $R^{2}$ ) of the variance. As can be seen in Step 3 in Table 3, VIQ is the only independent variable that makes a unique statistically significant contribution to the model, with an odds ratio of 1.10 .

[INSERT TABLE 3 ABOUT HERE] 
The frequency of the subtraction strategy was measured as the proportion of trials on which the subtraction strategy had been used. The regression analysis on the frequency data showed that the model accounted for $57 \%$ of the variance (Adjusted $\left.R^{2}=.56\right), F(3,116)=$ $51.81, p<.001$. As can be seen in Step 3 of Table 3, the only significant predictor in the model is again VIQ.

The adaptivity of the subtraction strategy use was measured by comparing the location of the actual change point (i.e., the numerosity on which a participant switched from the addition towards the subtraction strategy in the choice condition) with the location of the optimal change point as revealed by the no-choice data (Luwel et al., 2003). Since individuals are not completely systematic in their strategy choices (i.e., they sometimes use the subtraction strategy before the change point and the addition strategy after the change point), we defined the actual change point as the first numerosity on which participants started to use the subtraction strategy and did so for at least three consecutive numerosities (see Luwel et al., 2005). The optimal change point was determined by fitting a linear regression on the individual response-time patterns of the correctly solved trials of both nochoice/addition and no-choice/subtraction conditions (see Figure 2). The numerosity on which both regression lines intersect each other is considered as the optimal change point, since from this trial onwards the subtraction strategy becomes faster than the addition strategy without a loss of accuracy. The absolute difference in location between the actual and the optimal change point can be conceived as a measure of adaptivity: the smaller this difference, the better an individual's actual strategy choices are calibrated to his/her unbiased estimates of strategy performance. 


\section{[INSERT FIGURE 2 ABOUT HERE]}

The regression analysis on the difference scores revealed that the model accounted for $16 \%$ of the variance (Adjusted $R^{2}=.14$ ), $F(3,115)=7.28, p<.001$. As in most of the analyses, only VIQ made a statistically significant unique contribution to the model (see Step 3 in Table 3).

\subsection{Strategy execution}

The regression analysis on the no-choice solution times of correctly solved items indicated that the total variance accounted for by this model was $58 \%$ (Adjusted $R^{2}=.57$ ), $F(3,116)=52.65, p<.001$. As shown in Step 3 of Table 3, all three predictors played a significant role in the speed of the subtraction strategy.

With respect to the no-choice error rates (i.e., the absolute deviation between the given response and the actual numerosity), the regression analysis revealed that the model accounted for $26 \%$ of the variance (Adjusted $R^{2}=.24$ ), $F(3,116)=13.70, p<.001$. It was shown that, once again, only VIQ made a statistically significant contribution to the model (see Step 3 in Table 3).

\subsection{Follow-up analyses}

The different regression analyses indicated that PIQ only played a very limited role in participants' strategic competence. This could either mean that PIQ does not play a role in the strategic competence at all, or that it plays a role but does not explain an additional portion of the variance above and beyond VIQ. To find out which of both possibilities would hold, we conducted a hierarchical regression analysis on the different strategic 
competence parameters. Since arithmetic proficiency was a control variable, it was always entered first, followed by PIQ in a second step, and VIQ in a third and last step.

We observed that, after having controlled for arithmetic proficiency, the inclusion of PIQ into the model at Step 2 did lead to a significant increase in the amount of variance explained by the model for all parameters, except for accuracy (see Table 3). Thus, at Step 2, PIQ was a significant predictor for all parameters involving strategy selection as well as for the speed of the subtraction strategy. Including VIQ into the model at Step 3, however, reduced the contribution of PIQ to all parameters to non-significance, except for speed. We can thus conclude that PIQ does play a role in most of the strategic competence parameters but, except for speed, it does not explain a unique portion of the variance above and beyond VIQ.

\section{Discussion}

We observed that only VIQ plays a significant role in the parameters regarding the selection of the subtraction strategy (i.e., occurrence, frequency, and adaptivity). This finding can be explained by the fact that the frequent and, especially, adaptive use of this clever strategy necessitates a good insight in the task to determine which strategy works best on which problem. This kind of insight may be more strongly related to VIQ, which includes the abilities of abstract and numerical reasoning (as assessed by the similarities and arithmetic subtest, respectively), rather than to general visual-motor abilities captured by PIQ (Kaufman, 1994). VIQ was also a better predictor of strategy execution than PIQ, since VIQ significantly explained a unique portion of the variance of both the accuracy and the speed of the subtraction strategy, whereas PIQ only significantly predicted the speed of this strategy, but not its accuracy. A possible explanation for this finding is that the efficient 
execution of the subtraction strategy requires counting as well as elementary arithmetic skills (such as adding groups of blocks and subtracting the number of empty cells from the total number of cells in the grid) which are to some extent related to some of the abilities captured involved in the arithmetic subtest of the VIQ scale(Kaufman, 1994), even when controlling for arithmetic proficiency. As stated above, PIQ explains, in addition to arithmetic proficiency and VIQ, a unique portion of the variance in the speed but not in the accuracy of the subtraction strategy execution. Most probably, this is due to the involvement of processing speed, which is captured by the coding subtest which is part of the PIQ composite score (Kaufman, 1994). The lack of impact of PIQ in most parameters of strategic competence does not mean that PIQ is not involved in these parameters, rather it does not explain a unique portion of the variance above and beyond VIQ.

Obviously, the selection and execution of strategies in the present numerosity judgement task may involve specific abilities and skills that do not necessarily play a role in strategy use in other task domains, such as computational estimation. Therefore, it is recommendable to replicate the present findings in other task domains to determine the extent to which the contribution of a particular predictor is task-specific or, rather, underlying a general skill to select and execute strategies efficiently. Furthermore, there may be other variables which play an important role in individuals' strategy use, such as metacognition or self-regulation, and that should be taken into account in future work. Individuals with greater metacognitive or self-regulatory capabilities may have a better understanding of the demands of the task at hand as well as of the utility and appropriateness of various optional actions (Kuhn \& Pearsall, 1998; Luwel, Torbeyns, \& 
Verschaffel, 2003); this in turn may make them more prone to invent new (clever) strategies and to learn to apply them more efficiently and adaptively.

The present study may have important implications for mathematics education, since it indicates that the higher a child's VIQ score, the less instructional help it needs in discovering, executing, and adaptively selecting new solution strategies. Given this predictive value of VIQ for the quality of children's strategic performance, teachers can relatively easily identify those pupils who will probably experience most difficulties when being instructed to use multiple solution strategies in a flexible way. 


\section{References}

French, J. W., Ekstrom, R. B., \& Price, I. A. (1963). Kit of reference tests for cognitive factors. Princeton, NJ: Educational Testing Services.

Gaultney, J. F., Bjorklund, D. F., Goldstein, D. (1996).To be young, gifted and strategic: Advantages for memory performance. Journal of Experimental Child Psychology, 61, 43-66.

Geary, D. C., \& Brown, S. C. (1991). Cognitive addition: Strategy choice and speed-ofprocessing differences in gifted, normal, and mathematically disabled children. Developmental Psychology, 27, 398-406.

Georgas, D. D., Paraskevopoulos, I. N., Bezevegis, I. G., \& Giannitsas, N. D. (1997). Greek WISC-III: Wechsler intelligence scales for children. Athens: Ellinika Grammata. Hettinger-Steiner, H. (2006). A microgenetic analysis of strategic variability in gifted and average-ability children. Gifted Child Quarterly, 50, 62-74.

Kaufman, A. S. (1994). Intelligent testing with the WISC-III. New York: Wiley.

Kuhn, D., \& Pearsall, S. (1998). Relations between metastrategic knowledge and strategic performance. Cognitive Development, 13, 227-247.

Luwel, K., Foustana, A., Papadatos, Y., \& Verschaffel, L. (2011). The role of intelligence and feedback in children's strategy competence. Journal of Experimental Child Psychology, 108, 61-76.

Luwel, K., Lemaire, P., \& Verschaffel, L. (2005). Children's strategies in numerosity judgment. Cognitive Development, 20, 448-471. 
Luwel, K., Onghena, P., Torbeyns, J., Schillemans, V., \& Verschaffel, L. (2009). Strengths and weaknesses of the choice/no-choice method in research on strategy use. European Psychologist, 14, 351-362.

Luwel, K., Torbeyns, J., \& Verschaffel, L. (2003). The relation between metastrategic knowledge, strategy use and performance: Findings and reflections from a numerosity judgement task. European Journal of Psychology of Education, 18, 425-447.

Neter, J., Kutner, M. H., Nachtsheim, C. J., \& Wasserman, W. (1996). Applied linear regression models (3rd Ed.). London: Irwin.

Shallice, T. (1988). From neuropsychology to mental structure. Cambridge: Cambridge University Press.

Siegler, R. S. (1996). Emerging minds: The process of change in children's thinking. New York: Oxford University Press.

Siegler, R. S. (2005). Children's learning. American Psychologist, 60, 769-778.

Siegler, R. S., \& Lemaire, P. (1997).Older and younger adult's strategy choices in multiplication: Testing predictions of ASCM using the choice/no choice method. Journal of Experimental Psychology: General, 126, 71-92.

Wechsler, D. (1991). Manual for the Wechsler Intelligence Scale for Children ( $3^{\text {rd }}$ edition). San Antonio, TX: The Psychological Corporation. 


\section{Acknowledgement}

This study was funded within Op. Education by the European Social Fund, National Greek Resources, and GOA grant 2012/010 from the Research Fund K.U. Leuven, Belgium. 
Table 1

Descriptive Statistics of the Different Measures

\begin{tabular}{lcccc}
\hline Variable & $M$ & $S D$ & Min & Max \\
\hline FSIQ & 103.55 & 21.42 & 70 & 145 \\
VIQ & 106.12 & 21.63 & 55 & 145 \\
PIQ & 100.16 & 18.63 & 62 & 137 \\
Arithmetic Proficiency & 48.24 & 23.09 & 1.00 & 117 \\
Frequency & 26.99 & 30.33 & 0.00 & 100 \\
Adaptivity & 8.62 & 5.01 & 0.00 & 20.00 \\
No-Choice RT & 14.37 & 5.65 & 6.74 & 33.82 \\
No-Choice Error Rate & 0.84 & 1.00 & 0.00 & 4.58 \\
\hline Note: FSIQ = Full Scale IQ, VIQ = Verbal IQ, PIQ = Performance IQ & &
\end{tabular}


Table 2

Inter-correlations among the Primary Variables

\begin{tabular}{|c|c|c|c|c|c|c|}
\hline Variable & 1 & 2 & 3 & 4 & 5 & 6 \\
\hline 1. VIQ & - & - & - & - & - & - \\
\hline 2. PIQ & $.75^{\star \star}$ & - & - & - & - & - \\
\hline 3. Arithmetic Proficiency & $.74^{\star \star}$ & $.64^{* *}$ & - & - & - & - \\
\hline 4. Frequency & $.75^{\star *}$ & $.61^{* *}$ & $.61^{* *}$ & - & - & - \\
\hline 5. Adaptivity & $-.39^{* *}$ & $-.32^{* *}$ & $-.25^{\star}$ & $-.72^{\star \star}$ & - & - \\
\hline 6. No-Choice RT & $-.69^{* *}$ & $-.65^{\star \star}$ & $-.68^{\star \star}$ & $-.56^{\star \star}$ & .04 & - \\
\hline 7. No-Choice Error Rate &.$-49^{* *}$ & $-.40^{* *}$ & $-.46^{\star *}$ & $-.39^{* *}$ & $.18^{*}$ & $.46^{* *}$ \\
\hline
\end{tabular}

Note: Correlations are not reported for strategy repertoire since presence of the subtraction strategy was coded as a dichotomous variable. VIQ $=$ Verbal IQ, PIQ = Performance IQ, RT = Reaction Time.

$* p<.01 . * * p<.001$. 
Table 3

Results of the Hierarchical Regression Analyses for the Different Competence Parameters of the Subtraction Strategy

\begin{tabular}{|c|c|c|c|c|c|c|c|c|c|c|c|c|c|c|c|}
\hline \multirow[b]{2}{*}{ Variable } & \multicolumn{3}{|c|}{ Repertoire } & \multicolumn{3}{|c|}{ Frequency } & \multicolumn{3}{|c|}{ Adaptivity } & \multicolumn{3}{|c|}{ Solution Times } & \multicolumn{3}{|c|}{ Error Rates } \\
\hline & $\operatorname{Exp}(B)$ & Wald $\chi^{2}(1)$ & $p$ & Beta & $t(116)$ & $p$ & Beta & $t(115)^{\mathrm{a}}$ & $p$ & Beta & $t(116)$ & $p$ & Beta & $t(116)$ & $p$ \\
\hline Arithmetic & 1.10 & 29,71 & $<.001$ & .61 & 8.36 & $<.001$ & -.25 & -2.82 & .006 & -.69 & -10.42 & $<.001$ & -.46 & -5.60 & $<.001$ \\
\hline \multicolumn{16}{|l|}{ Step 2} \\
\hline Arithmetic & 1.06 & 11.22 & .001 & .36 & 4.03 & $<.001$ & -.08 & -.69 & .494 & -.113 & -5.66 & $<.001$ & -.339 & -3.169 & $<.001$ \\
\hline $\mathrm{PIQ}$ & 1.07 & 11.49 & .001 & .38 & 4.24 & $<.001$ & -.27 & -2.35 & .020 & -.356 & -4.37 & $<.001$ & -.184 & -1.72 & .09 \\
\hline \multicolumn{16}{|l|}{ Step 3} \\
\hline Arithmetic & 1.03 & 1.50 & .221 & .09 & .95 & .34 & .10 & .78 & .44 & -.34 & -3.62 & $<.001$ & -.20 & -1.60 & .11 \\
\hline $\mathrm{PIQ}$ & 1.02 & 0,51 & .475 & .09 & .97 & .34 & -.08 & -.57 & .57 & -.23 & -2.38 & .02 & .09 & 0.07 & .34 \\
\hline $\mathrm{VIQ}$ & 1.10 & 13.70 & $<.001$ & .62 & 5.64 & $<.001$ & -.41 & -2.71 & .008 & -.28 & -2.57 & .01 & .62 & 5.64 & $<.001$ \\
\hline
\end{tabular}

Note $:$ VIQ $=$ Verbal IQ, PIQ = Performance IQ, RT $=$ Reaction Time.

${ }^{\mathrm{a}}$ The degrees of freedom for adaptivity are 115 instead of 116 since it was not possible to determine the optimal change point for one participant. 


\section{Figure Captions}

Figure 1. Example of two stimuli that typically elicit the use of the addition strategy (a) and the subtraction strategy (b).

Figure 2. Example of two individual response-time patterns from a no choice/addition and a no-choice/subtraction condition respectively with their corresponding linear regression lines 

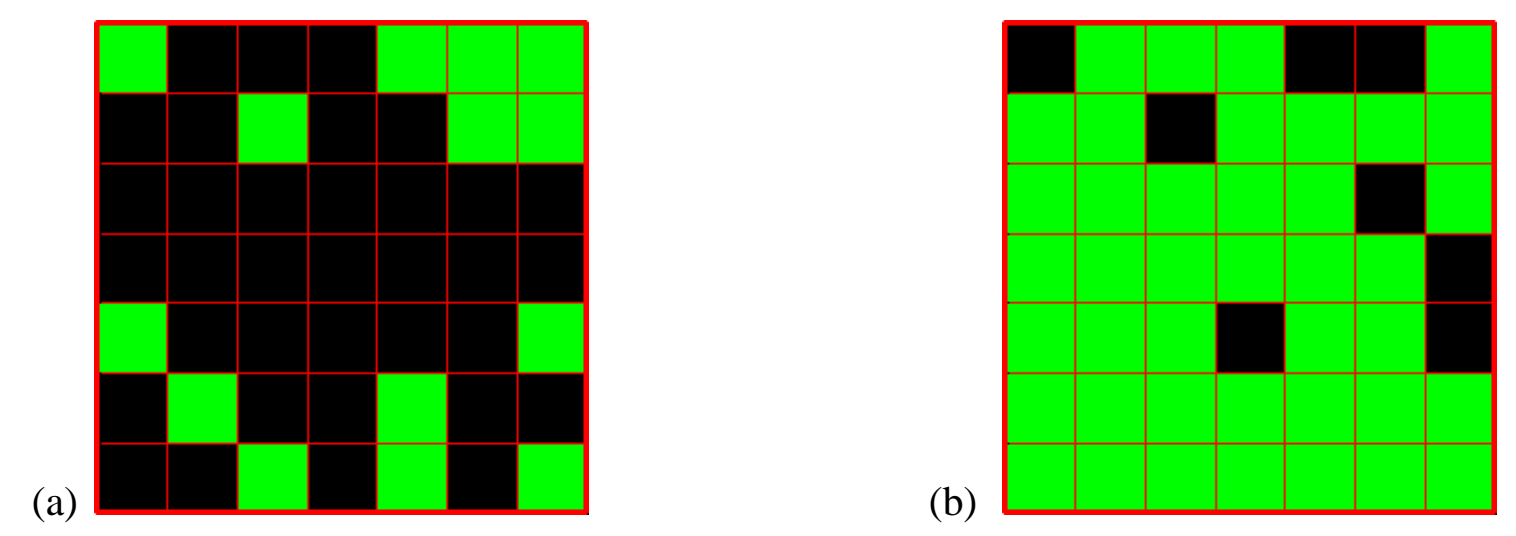


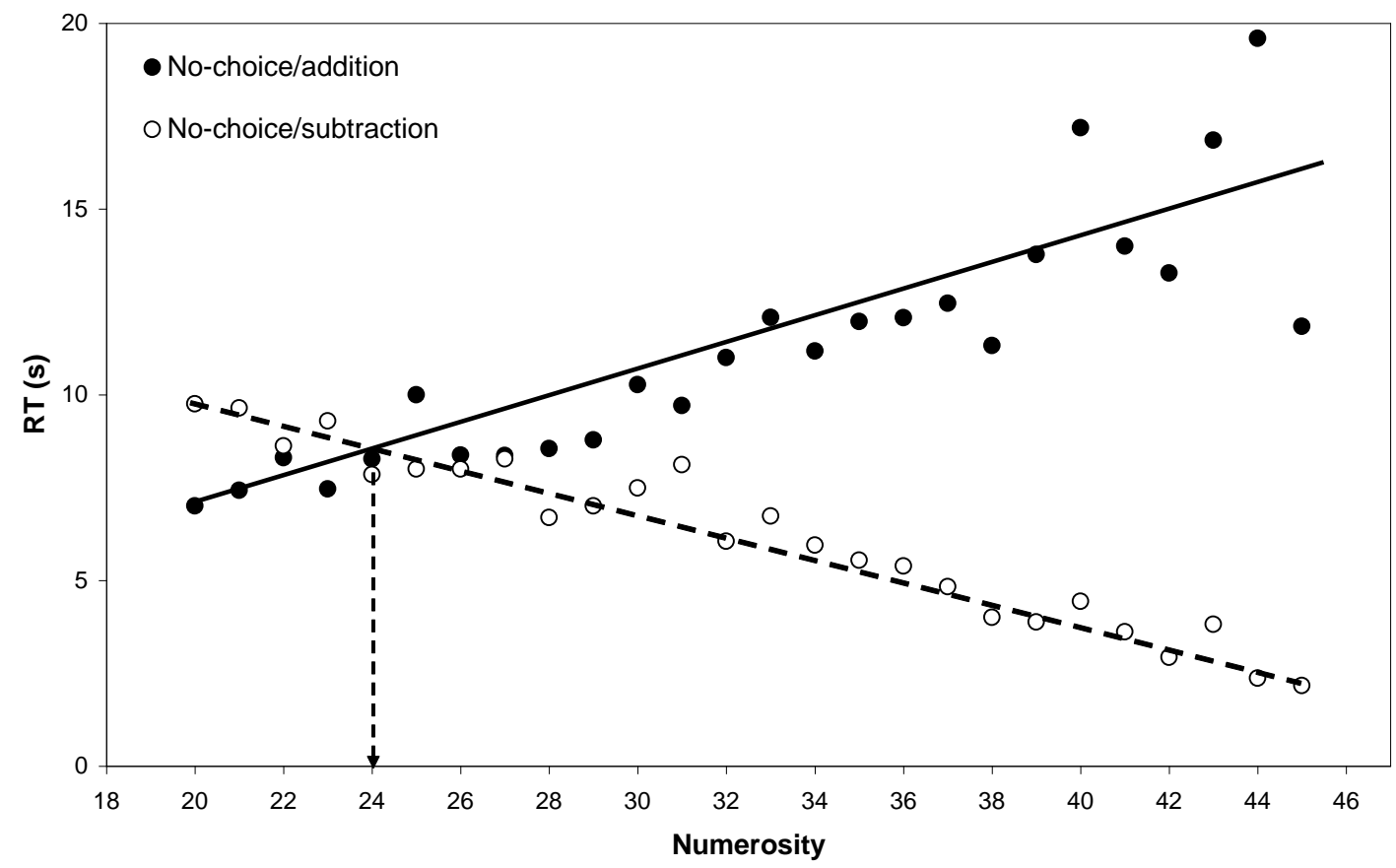

